\title{
SELECTION OF DEFUZZIFICATION METHOD TO OBTAIN CRISP VALUES FOR REPRESENTING UNCERTAIN DATA IN A MODIFIED SWEEP ALGORITHM
}

\author{
Oleh : \\ Gunadi W. Nurcahyo \\ Postgraduate Studies, Universitas Putera Indonesia "YPTK" Padang \\ gunadiwidi@yahoo.co.id
}

\begin{abstract}
A study of using fuzzy-based parameters for solving public bus routing problem with uncertain demand is presented. The fuzzy-based parameters are designed to provide data required by the route selection procedure. The uncertain data are represented as linguistic values which are fully dependent on the user's preference. Fuzzy inference rules are assigned to relate the fuzzy parameters to the crisp values which are concerned in the route selection process. This paper focuses on the selection of the Defuzzification method to discover the most appropriate method for obtaining crisp values which represent uncertain data. We also present a step by step evaluation showing that the fuzzy-based parameters are capable to represent uncertain data replacing the use of exact data which common route selection algorithms usually use.
\end{abstract}

Keywords : public bus routing, route selection, fuzzy-based parameter, defuzzification.

\begin{abstract}
A study of using fuzzy-based parameters for solving public bus routing problem with uncertain demand is presented. The fuzzybased parameters are designed to provide data required by the route selection procedure. The uncertain data are represented as linguistic values which are fully dependent on the user's preference. Fuzzy inference rules are assigned to relate the fuzzy parameters to the crisp values which are concerned in the route selection process. This paper focuses on the selection of the Defuzzification method to discover the most appropriate method for obtaining crisp values which represent uncertain data. We also present a step by step evaluation showing that the fuzzy-
\end{abstract}

based parameters are capable to represent uncertain data replacing the use of exact data which common route selection algorithms usually use.

Keywords: public bus routing, route selection, fuzzy-based parameter, defuzzification.

\section{INTRODUCTION}

This paper is based on a case study on a public transport company located in Semarang, Indonesia (Gunadi, et al., 2002). We propose a solution to public bus routing problem concerning uncertain demand, travel distance, and type of road. The objective is to perform route selection 
using a modified search technique that is capable to capture uncertain data. A model called Sweep algorithm has been introduced previously for solving route selection problem for goods delivery vehicles (Gillet and Miller, 1974). This algorithm performs a route selection using exact data and certain demand. Teodorovic and Pavkovic (1995) apply the sweep algorithm for solving route selection when demand is uncertain with capacity constraint and additional distance is allowed. In our case, the algorithm is applied to public bus routing problem when uncertain demand is a major concern and no additional distance is allowed. Fuzzybased parameters are proposed to represent the uncertain data. The fuzzy inference rules are assigned in the fuzzy system to provide data used in the modified sweep algorithm.

This paper focuses on the defuzzification part of the proposed solution where the crisp values are obtained and concerned in the route selection. Defuzzification is a reverse process of Fuzzification. Basically, defuzzification is a mapping from a space of fuzzy control actions defined over an output universe of discourse into a space of non fuzzy (crisp) control actions (Rao \& Saraf, 1995). Defuzzification is a decision making process in a fuzzy set. It is a selection process that transforms a fuzzy set to a scalar such that the scalar is the representative identity of the support set of the given fuzzy set (Roychowdhury et al., 1994). Defuzzification is defined by Runkler (1996) as a mapping of fuzzy sets $\tilde{A}$ to significant elements with respect to $\tilde{A}$, denoted as $F^{-1}: F(X) \rightarrow X$. Lim et al. (1998) expressed defuzzification by: $y_{o u}=$ defuzzifier(y), where $y$ is the fuzzy control action, $y_{o u}$ is the crisp control action; and the defuzzifier is the defuzzification operator.

\section{PROBLEM DEFINITION}

A route selection for public bus routing problem is performed concerning demand, travel distance and type of road. Demand is the major concern and in the case of searching for new routes, demand is uncertain. This paper discusses the three parameters required in a route selection process where data are uncertain or, in other words, the data cannot be represented as exact numbers. For known locations of nodes and links in which public bus service does not exist, three conditions of links must be identified approximately and represented as linguistic value such as: very low, low, medium, high, bad, good, etc. The linguistic values are determined based on the user's preference. The conditions of links which become the parameters are namely: Demand, Travel Distance, and Type of Road. Fuzzy inference rules are assigned to convert linguistic values of data into crisp values. The crisp values which represent three parameters are used as the weights of the candidate links. By using the fuzzy-based parameters, the proposed system is designed to capture uncertain data and use them in the route selection process.

\section{MODIFIED SWEEP ALGORITHM (MSA)}

Modification is made to the second stage of the original sweep algorithm, i.e. Route Generation. In the first stage, clustering process is performed using the same procedure as used in the original sweep algorithm. A node is joined with its nearest neighbour based on its polar coordinate angle and the capacity of each vehicle. The objective of clustering in the modified sweep algorithm is to provide a procedure which enables user to easily group the available nodes and determine which 
cluster a node or link should be attached to. Node [0] is assumed to be the centre polar coordinate.

Since node [0] of the real data is located in coordinate $x=324$ and $y=124$, we reformulate the calculation of polar coordinate angles. If the location of node [0] is denoted by $x_{0, y_{0}}$ then the location of node [i] is defined as $x_{i}-x_{0}, y_{i}-y_{0}$. The polar coordinate angle is defined using sinus function to suit the functions provided by Pascal programming language that is used in the experiment:

\section{$\operatorname{Sin} \alpha_{i}=\left(y_{i}-y_{0}\right) /$ radian}

where radian is defined by:

$$
\operatorname{radian}=\sqrt{ }\left(\left(\mathrm{x}_{\mathrm{i}}-\mathrm{x}_{0}\right)^{2}+\left(\mathrm{y}_{\mathrm{i}}-\mathrm{y}_{0}\right)^{2}\right)
$$

After clustering process is completed, we have to analyse the list of links in each cluster. As mentioned previously, clustering process enables us to group the links and perform the route selection. The result may not satisfy the user because of reasons such as: the links attached in a cluster generate a very short route, or there are too many clusters. In the case of unsatisfactory clusters, we can make changes by replacing or deleting links in a cluster, and combining two clusters. When all clusters are set, we have to determine the start and end node in each cluster. In this case, start and end node are the nodes where the bus stations are located.

In the second stage, a thorough modification is made by replacing travelling salesman (TSP) route selection method with a weighted-directed search (WIDI). Unlike TSP which selects routes by combining nodes based on short distance, WIDI selects routes by combining links based on demand as explained in
Section 5. Comparisons between SA and MSA are presented in Section 7.

In selecting a link to be attached to the generated route, there are several constraints that are considered. Assuming that $l[i, j]$ is a link connecting node $i$ to node $j$ and $L$ is a set of links from start node node[1] to end node node [n], link $l[i, j] \in L$ if:
a. weight $[i, j]>=$ weight $[i, j+1]$
where: $0 \leq \mathrm{i} \leq$ MaxNode
$0 \leq \mathrm{j} \leq$ MaxNode
b. node [i] and node[j] are predecessors of node [n]
c. $i \neq$ node $[n]$ and $j \neq$ node $[n-1]$

The WIDI Graph Search algorithm is described as follows:

1. Initialize Node[1]=Start_Node, Destination=End_Node, Maximum node $=$ Max_Node,$\quad m=$ maximum adjacent nodes, and $x \in\{$ Node[1], Node[2], ... Node[n]\}.

2. Store Node[1] in the first list of nodes.

3. For $i=1$ to Max_Node do

4. $x=$ Node $[i]$.

5. For $j=1$ to $m$ do

6. Find an adjacent node to $x$ in the weight matrix.

7. If found and the adjacent node is not in the list of nodes and not in the Adjacent List then store the adjacent node in the Adjacent List.

8. Next $j$

9. Next $i$

10. Initialize $y=$ Destination, $t=$ false

11. While $y \neq$ Start_Node do

12. for $i=1$ to Max_Node do

13. for $j=1$ to $m$ do

14. if $\operatorname{Node}[i, j]=\mathrm{y}$ then store Node $[i]$ and Node $[i, j]$ in the track, $t=$ true.

15. $\operatorname{next} j$ 
16. if $t=$ true then set the next destination $y=\operatorname{Node}[\mathrm{i}]$

17. next $i$

18. End while

19. Set an empty stack to store generated links.

20. Start route searching from the Start_Node to End_Node. Initialize $s=$ Start_Node and push to the stack.

21 . If $s$ has more then one adjacent nodes then check whether those adjacent nodes are in the same track or not. If not go to step 23 .

22. If yes then compare the weights of those nodes and select the node with highest weight.

23. Push the node to the stack.

24. Set $s$ to the selected node.

25. Repeat step 21 until $s=$ End_Node.

26. The sequence of nodes in the stack represents the links of the selected route and this is the result of the route searching process. To search for another route, repeat step 10 .

\section{FUZZY PARAMETERS}

It is perceived that a candidate route is selected based on three parameters. These parameters coincide with the factors that are used by the company in route selection. Below are the parameters of the candidate routes. Note that each parameter has a range of value from zero (0) to one (1).

a. Demand (D)

Demand is the most concerned parameter of the company in selecting a route. In this research, demand is combined with the other attributes as linguistic data for the route selection process. There are 5 membership functions for demand that show the degree of potential demand: VERY HIGH, HIGH, MEDIUM, LOW, and VERY LOW denoted as VH, H, M, L, and VL.
Demand at each node is only approximately known and represented as a triangular fuzzy number because this membership representation shows minimum and maximum boundary clearly. Figure 1 shows the membership function of triangular fuzzy number $\boldsymbol{D}$ representing demand at the link. Triangular fuzzy number $\boldsymbol{D}=\left(d_{1}, d_{2}, d_{3}\right)$ is described by its left boundary $d_{1}$ and its right boundary $d_{3}$. Thus, the decision maker can estimate that demand at the node will not less than $d_{1}$ or greater than $d_{3}$ based on his experience and intuition or available data. Figure 1 shows the fuzzy sets of linguistic data for demand.

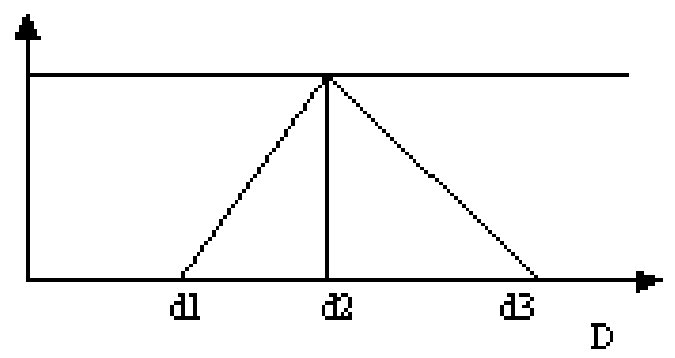

Fig. 1. Triangular Fuzzy Number $D$

It can be seen in Figure 1 that each value has left and right boundary. For example, right boundary of VL is 50 . The grade of membership of VL at this point is zero. This means that when demand is 50 or greater, the appropriate linguistic data for demand is Low. At the same point, the grade of membership of $L$ is 1 . Hence, if demand is 50 then the linguistic value of demand is LOW. Figure 2 shows the complete membership functions of demand.

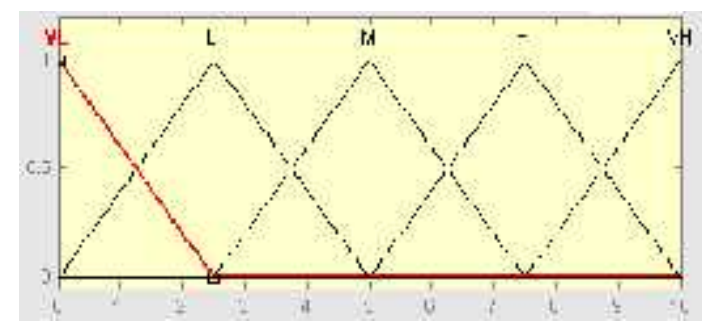


Fig. 2. Demand Membership Functions

b. Travel Distance (TD)

Distance of a route is concerned because it is related operational cost. A shorter route requires less operational cost. In public bus routing, a short distance route with minimum cost cannot be selected when demand is low. Therefore, travel distance cannot be used as single parameter for public bus route selection. The linguistic data of travel distance consists of VERY_LONG_DISTANCE (VL), LONG_DISTANCE MEDIUM DISTANCE SHORT_DISTANCE (SS), and VERY_SHORT_DISTANCE (VS). The membership functions of this parameter are shown in Figure 3. The triangular fuzzy number is noted by $T D=(t d 1, t d 2, t d 3)$.

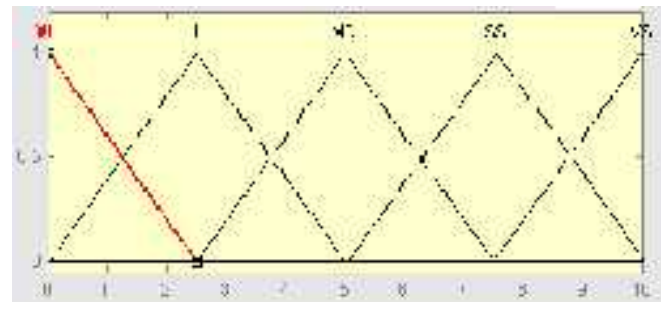

Fig. 3. Distance Membership Functions

c. Type of Road (TR)

Type of road includes the physical condition of the roads in a route. A road with good condition enables the buses to minimize travel time. Conversely, a bad road may increase travel time and, in sequence, increases operational cost. Type of road consists of three conditions of road denoted by BAD, AVERAGE, and GOOD with membership functions as shown in
Figure 4. Notation for triangular fuzzy number is $T R=(\operatorname{tr} 1, \operatorname{tr} 2, \operatorname{tr} 3)$.

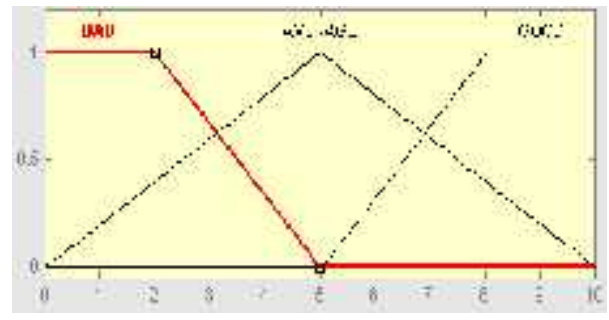

Fig. 4. Type of Road Membership Functions

\section{FUZZY RELATION}

In this section, we illustrate the relation between the initiated fuzzy input and the required output. Figure 5 shows the whole structure of the proposed solution consisting of input, reasoning rules, and output. Three inputs are captured consisting of demand, distance, and type of road as described previously.

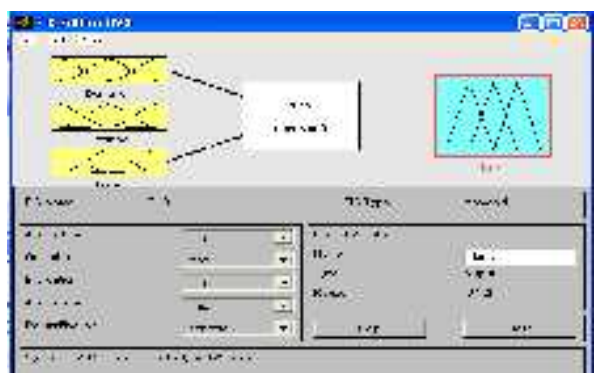

Fig. 5. Fuzzy System Structure

The three inputs are denoted as:

$\mu_{A}(D)$ where $A=\{V L, L, M, H, V H\}$

(1)

$\mu_{B}(T D)$ where $B=\{V L, L L, M D, S S, V S\}$

(2)

$\mu_{C}(T R)$ where $C=$

$\{B A D, A V E R A G E, G O O D\}$

The inference rules relate these input to the output Link consisting of seven 
membership functions i.e. NEGATIVE LARGE (NE), NEGATIVE MEDIUM (NM), NEGATIVE SMALL (NS), ZERO (ZE), POSITIVE SMALL (PS), POSITIVE MEDIUM (PM), and POSITIVE LARGE (PL). In this paper, we use Mamdani type Fuzzy Inference Rules. As noted by Mamdani and Assilian (1975), every rule represents a fuzzy relation. In our case, every rule represents relation between the three inputs to Link. Figure 6 shows the complete membership functions of Link which is denoted as:

\section{$\mu_{O}($ Link) $\quad$ where $\{N L, N M, N S, Z E, P S, P M, P L\}$}

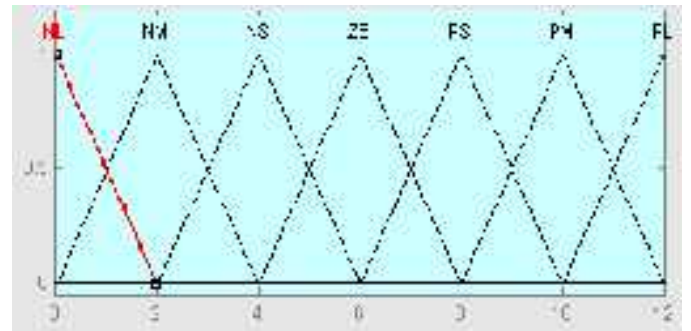

Fig. 6. Link Membership Function

Let us denote by $R$ the fuzzy relation between $D, T D, T R$, and Link. As denoted in Teodorovic and Pavkoviv (1996), membership function $\mu_{R}(x, y)$ of this fuzzy relation equals:

$\mu_{R}(x, y)=\min \left\{\mu_{A}(x), \mu_{B}(y)\right\} \quad \forall x, y$

In this paper, we denote the fuzzy relation as:

$\mu_{R}(x, y)=\min \left\{\min \left(\mu_{A}(D), \mu_{B}(T D), \mu_{C}(T R)\right)\right.$, $\left.\mu_{O}(\operatorname{Link})\right\} \forall x, y$

where $x$ is input and $y$ is output.
The following rules show the fuzzy relation and are graphically shown in Figure 7: If [Demand is M] and [Distance is SS] and [Type is BAD] Then [Link is NM] If [Demand is M] and [Distance is SS] and [Type is AVERAGE] Then [Link is $\mathrm{ZE}]$

If [Demand is M] and [Distance is SS] and [Type is GOOD] Then [Link is ZE]

The fuzzy relations of those rules are shown as follow:

$$
\begin{aligned}
& \mu_{R}(x, y)=\min \left\{\operatorname { m i n } \left(\mu_{M}(D), \quad \mu_{S S}(T D)\right.\right. \text {, } \\
& \left.\left.\mu_{B A D}(T R)\right), \mu_{N M}(\operatorname{Link})\right\} \\
& \mu_{R}(x, y)=\min \left\{\operatorname { m i n } \left(\mu_{M}(D), \quad \mu_{S S}(T D),\right.\right. \\
& \left.\left.\mu_{A V E R A G E}(T R)\right), \mu_{Z E}(\operatorname{Link})\right\} \\
& \mu_{R}(x, y)=\min \left\{\operatorname { m i n } \left(\mu_{M}(D), \quad \quad \mu_{S S}(T D),\right.\right. \\
& \left.\left.\mu_{G O O D}(T R)\right), \mu_{Z E}(\operatorname{Link})\right\}
\end{aligned}
$$

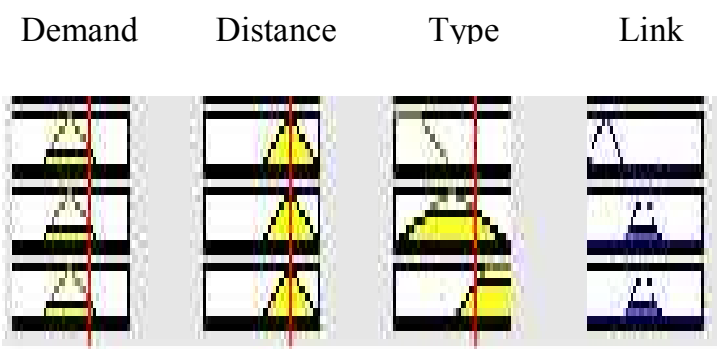

Fig. 7. Fuzzy Relations

\section{DEFUZZIFICATION METHODS}

Defuzzification is the most important part that influences the performance of the fuzzy systems. The task of defuzzification module is to find a single crisp value that summarizes the fuzzy set that defines the fuzzification (Rao \& Saraf, 1995). The defuzzification technique is aimed at producing a non fuzzy control action that best represents the possibility distribution of an inferred fuzzy control action (Kung \& Liaw, 1994). The defuzzification techniques are described as follow.

\section{Center of Area/Gravity}


It is one of the most commonly used defuzzification techniques. This method determines the centre of the area of the combined membership functions (Rao \& Saraf, 1995). Runkler (1996) calculated the centroid or centre of gravity (COG) of the area under the membership function as:

$$
F^{-1} \operatorname{cog}(\tilde{A}):=\int_{x} \mu \tilde{A}(x) \cdot x d x
$$

$$
\int_{x} \mu \tilde{A}(x) d x
$$

\section{Maxima Methods}

COG is a defuzzification method regarding the area under the membership function. Maxima methods consider values with maximum membership (Runkler, 1996). There are different maxima methods with different conflict resolution strategies for multiple maxima, e.g., first of maxima (FOM), last of maxima LOM), mean of maxima (MOM), and centre of maxima (median). Rao and Saras (1995) distinguish those methods as the following. First of maxima uses the union of the fuzzy sets and takes the smallest value of the domain with maximal membership degree. Last of Maxima uses the union of the fuzzy sets and, on the other hand, takes the largest value of the domain with maximal membership degree. Middle of Maxima is similar to first of maxima and last of maxima. Instead of determining the defuzzified value to be the first or the last from all values, this method takes average of these two values. We use MOM and LOM as the representation of maxima methods which are denoted as follow. Mean of Maxima:

$$
\mathrm{U}=\sum^{\mathrm{R}} \mathrm{u}_{\mathrm{i}} / \mathrm{R}
$$

(8) $i=1$

Last of Maxima:

$$
\begin{aligned}
& \mathrm{U}=\sup \left(\mathrm{u}^{\prime}\right), \mu\left(\mathrm{u}^{\prime}\right)=\quad \sup \quad \mu(\mathrm{u}) \\
& \mathrm{u}=\in[\text { Min,Max }]
\end{aligned}
$$

\section{EXPERIMENTAL RESULTS}

Route selections are performed using both exact and fuzzy using the Modified Sweep Algorithm. There are 27 nodes included in the network and data are only known approximately. The connected links are shown in Appendix A that also consists of information related to the given links. The selection task aims to generate the best routes which offer maximum demand. Weights of links which represent demand, distance, and type of road are concerned in the route selection. Mamdani type Fuzzy Inference System is used to relate input parameters to the output parameter. This section also evaluates three defuzzification methods i.e. Centroid, Mean of Maxima (MOM) and Last of Maxima (LOM) in performing the conversion of linguistic values into crisp values. The three defuzzification methods are evaluated to select the best method to be applied in the algorithm.

The experiment is conducted to investigate the feasibility of fuzzy system in providing data for route selection process. The experiment aims to find evidences that linguistic values representing uncertain data are capable for replacing exact data in public bus routing problem. We evaluate the parameters one by one starting from the most concern parameter i.e. demand. A route is selected based on the number of demand in each link. The secondconcerned parameter is distance. When two candidate links have approximately the same demand, distance parameter is 
evaluated to identify a link with shorter distance. Note that a candidate link with longer distance may be selected if it has higher number of demand. Type of road is concerned because this parameter is related to operational cost and travel time. Type of road is physical condition of road. A bad condition of road may increase operational cost and travel time. We evaluate the parameters one by one to study their behaviour.

The experiment is conducted to investigate the feasibility of fuzzy system in providing data for route selection process. The experiment aims to find evidences that linguistic values representing uncertain data are capable for replacing exact data in public bus routing problem. We evaluate the parameters one by one starting from the most concern parameter i.e. demand. A route is selected based on the number of demand in each link. The secondconcerned parameter is distance. When two candidate links have approximately the same demand, distance parameter is evaluated to identify a link with shorter distance. Note that a candidate link with longer distance may be selected if it has higher number of demand. Type of road is concerned because this parameter is related to operational cost and travel time. Type of road is physical condition of road. A bad condition of road may increase operational cost and travel time. We evaluate the parameters one by one to study their behaviour.

\section{One Parameter}

We have previously performed the experiment using parameter demand consisting of exact data using MSA. In this experiment, MSA is applied using the same parameter consisting of fuzzy data. The objective is to evaluate the capability of fuzzy data in replacing exact data of demand for route selection. Two fuzzy sets are presented to illustrate the possibility of two different user's preferences. The results of this experiment are compared with the previous results of exact demand data as shown in Figure 8 for alternative links. It is shown in Figure $8 \mathrm{a}$ that the fuzzy data pattern may follow the exact data pattern (the weight line). Figure $8 b$ shows only the fuzzy data pattern consisting Centroid, MOM and LOM defuzzification methods.

a)

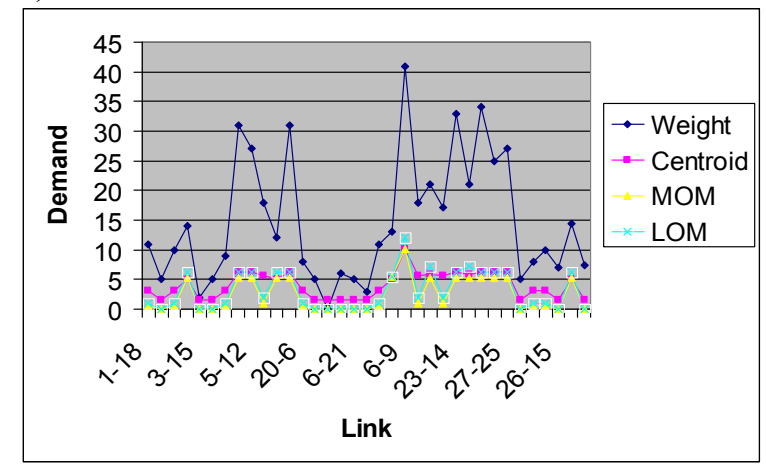

b)

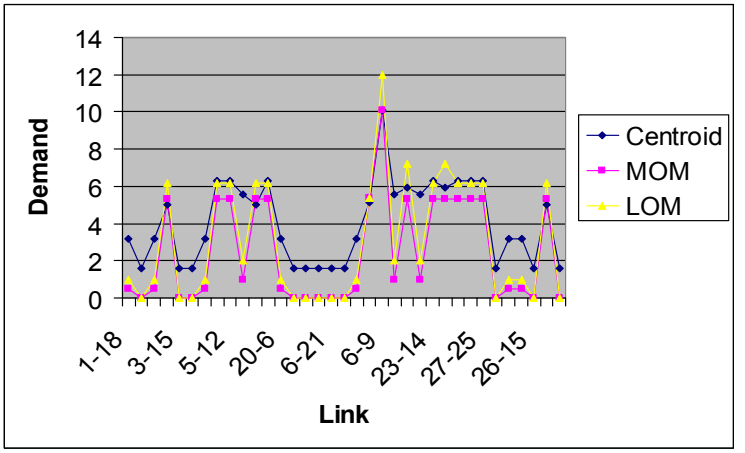

Figure 8: Demand Patterns

\section{Two Parameters}

Based on the experiment using one parameter, it is concluded that fuzzy data is capable to replace exact data indicated by the similar data pattern as shown in Figure 8. In this section, we present the next experiment based on the same case using additional parameter Distance. The use of parameter Demand and Distance in this experiment aim to obtain more accurate results which represent the real condition 
of public bus routing problem. The objective of this experiment is to evaluate the capability of fuzzy system in providing weight of link required by the MSA. The weight data represents the linguistic values of both Demand and Distance. The fuzzy relations between Demand, Distance, and Link are presented in Fuzzy Associative Matrix (FAM) as shown in Figure 9. FAM shows 25 rules relating the fuzzy input to the output. The complete results are shown graphically in appendix B.
In the first six results, i.e. demand $\leq 5$, Centroid, MOM and LOM methods have the same data pattern. At certain point, the graph raises drastically separating the lower parts with the higher parts. When demand $\geq 6$ the results of MOM and LOM still follow the same pattern. The results of Centroid, on the other hand, move to different pattern where there are more frequent changes of crisp values. This type of data pattern is more representative for providing weight data required by the proposed model.

Travel Distance

\begin{tabular}{|c|c|c|c|c|c|}
\hline & VL & $\mathbf{L L}$ & MD & SS & VS \\
\hline VL & NL & NL & NL & NL & NM \\
\hline $\mathbf{L}$ & MN & $\mathbf{N M}$ & $\mathbf{N M}$ & $\mathbf{N S}$ & NS \\
\hline $\mathbf{M}$ & NS & $\mathbf{Z E}$ & $\mathbf{Z E}$ & $\mathbf{Z E}$ & $\mathbf{Z E}$ \\
\hline H & PS & PS & PS & $\mathbf{P M}$ & PM \\
\hline VH & $\mathbf{P M}$ & $\mathbf{P M}$ & PL & PL & PL \\
\hline
\end{tabular}

Figure 9: Fuzzy Associative Matrix

It is shown in Figure 8 that, in general, the crisp value increases as the value of Demand and Distance membership functions increase. Note that the value of Distance membership functions does not represent the actual distance. As shown in the order of fuzzy set Link (see notation 4), the leftmost membership function represents the longest travel distance and vice versa. The Distance parameter is required to select a link when two candidate links have approximately the same demand. In our case, short distance is not a major concern. A candidate link may be selected even if it has longer distance than the other candidate. Let us see the following results:

Result 1:

$=9.01$

$$
\mu_{M}(D)=10, \mu_{S S}(T D)=15, \mu_{Z E}(\operatorname{Link})
$$

Result 2:

$$
\mu_{H}(D)=15, \mu_{L L}(T D)=5, \mu_{P S}(\operatorname{Link})
$$


Both results represent two candidate links. Result 1 is a link that has medium demand and short distance. Result 2, on the other hand, is a link that has high demand and long distance. In this case, although it has longer distance than result 1 , result 2 is selected because it has higher demand.

\section{Three Parameters}

As shown previously, two parameters containing five membership functions each require 25 rules in the fuzzy inference system. When we put one more parameter containing three membership functions, there will be 75 rules relating the parameters with the output Link. In using three parameters, we only present the results of the Demand membership functions $\mathrm{VL}, \mathrm{M}$, $\mathrm{VH}$, and Distance membership functions VL, MD, and VS to represent the final results as shown graphically in the following figures.

It is obvious that the results of this experiment share the same pattern as those of the previous experiment. The Centroid method also produces more representative results than MOM and LOM because there is a gradual increase of crisp values for certain membership function of Demand and Distance. Table 2 lists several instances of fuzzy results using three parameters as graphically shown in appendix C. By concerning parameter Type of Road, the MSA is capable to avoid bad links even if those links have higher number of demand. Let us compare case 14 and case 22 for example. Although the demand of case 22 is very high, case 14 is better because it has a better condition of road even if the demand is medium. A better link is indicated by the higher crisp value of parameter Link.

Table 2: Fuzzy Results using Three Parameters

\begin{tabular}{|c|c|c|c|c|}
\hline CASE & DEMAND & DISTANCE & TYPE & LINK \\
\hline 1 & \multirow{9}{*}{ Very Low } & \multirow{3}{*}{ Very Long } & $\mathrm{Bad}$ & 0.632 \\
\hline 2 & & & Average & 0.632 \\
\hline 3 & & & Good & 0.632 \\
\hline 4 & & \multirow{3}{*}{ Medium } & $\mathrm{Bad}$ & 0.632 \\
\hline 5 & & & Average & 0.632 \\
\hline 6 & & & Good & 0.632 \\
\hline 7 & & \multirow{3}{*}{ Very Short } & $\mathrm{Bad}$ & 0.632 \\
\hline 8 & & & Average & 2.02 \\
\hline 9 & & & Good & 2.02 \\
\hline 10 & & & $\mathrm{Bad}$ & 0.632 \\
\hline
\end{tabular}




\begin{tabular}{|c|c|c|c|c|}
\hline 11 & \multirow{8}{*}{ Medium } & \multirow[t]{2}{*}{ Very Long } & Average & $\begin{array}{l}4.01 \\
4.01\end{array}$ \\
\hline 12 & & & & 4.01 \\
\hline 13 & & \multirow{3}{*}{ Medium } & Bad & 2.02 \\
\hline 14 & & & Average & 6.01 \\
\hline 15 & & & Good & 6.01 \\
\hline 16 & & \multirow{3}{*}{ Very Short } & $\mathrm{Bad}$ & 2.02 \\
\hline 17 & & & Average & 6.01 \\
\hline 18 & & & Good & 6.01 \\
\hline 19 & \multirow{9}{*}{ Very High } & \multirow{3}{*}{ Very Long } & $\mathrm{Bad}$ & 4.01 \\
\hline 20 & & & Average & 10 \\
\hline 21 & & & Good & 10 \\
\hline 22 & & \multirow{3}{*}{ Medium } & $\mathrm{Bad}$ & 4.01 \\
\hline 23 & & & Average & 11.4 \\
\hline 24 & & & Good & 11.4 \\
\hline 25 & & \multirow{3}{*}{ Very Short } & $\mathrm{Bad}$ & 4.01 \\
\hline 26 & & & Average & 11.4 \\
\hline 27 & & & Good & 11.4 \\
\hline
\end{tabular}

\section{Conclusions}

This paper proposes a solution to public bus routing problem when demand is uncertain using the Modified Sweep Algorithm. The route selection is performed concerning three parameters i.e. Demand, Distance, and Type of Road. The parameters are presented in linguistic value indicating the degree of membership functions. Fuzzy inference rules are assigned to relate the parameters to the Link membership functions. Centroid method is chosen to perform the defuzzification process based on the comparison between this method and another well-known defuzzification method called Maxima method which is represented by MOM and LOM methods. Centroid defuzzification method converts the Link membership functions into crisp values. The crisp values are assigned as the weights of the candidate links which are selected by the algorithm to generate route. A selected link is indicated by higher crisp value than another candidate.

The experiment shows a correlation between parameters concerned in the route selection and the weights of the candidate links. A weight represents a condition of link based on the three parameters. We can adjust the weight depending on our preference of a certain link. A change of value of a parameter may produce a different crisp value. Therefore, before performing the route selection, we have to be certain that the membership functions of the parameters are set correctly and representatively.

\section{References}

B. E. Gillet, L. R. Miller, A heuristic algorithm for the vehicle dispatch problem, Operation Research, vol. 22, pp. 340-349, 1974.

D. H. Rao, S. S. Saraf, Study of Defuzzification Methods of Fuzzy Logic Controller for Speed Control of a DC 
Motor, IEEE Transactions, pp. 782-787, 1995.

D. Teodorovic, G. Pavkovic, A Simulated Annealing Technique Approach to the Vehicle Routing Problem in the Case of the Stochastic, Transportation Planning Technology, vol. 16, pp. 261-273, 1992.

D. Teodorovic, G. Pavkovic, "The fuzzy set theory approach to the vehicle routing problem when demand at nodes is uncertain", Fuzzy Sets and Systems, vol. 82, pp. 307-317, 1996.

E. Mamdani, S. Assilian, An experiment in linguistic synthesis with a fuzzy logic controller, Internat. J. Man-Machine Studies vol. 7, pp. 1-13, 1975.

G. J. Klir, B. Yuan, Fuzzy Sets and Fuzzy Logic: Theory and Applications, Prentice Hall PTR, New Jersey, 1995.

G. J. Klir, U. H. St. Clair, and B. Yuan, Fuzzy Set Theory: Foundations and Applications, Prentice Hall, Upper Saddle River, NJ, 1997.

Gunadi W. Nurcahyo, Siti Mariyam Shamsuddin, Rose Alinda Alias, M.
Noor Md. Sap, Vehicle Routing Problem for Public Transport: A Case Study, International Technical Conf. on Computers/Systems, Circuits and Communications, vol. 2, pp. 1180-1183, Phuket, Thailand, 16-19 July 2002.

S. Kagaya, S. Kikuchi, R. A. Donnelly, Use of a Fuzzy Theory Technique for Grouping of Trips in the Vehicle Routing and Scheduling Problem, European Journal of Opl. Res., vol. 76, pp. 143154, 1994.

T. A. Runkler, Extended Defuzzification Methods and Their Properties, IEEE Transactions, pp. 694-700, 1996.

T. A. Runkler, Selection of Appropriate Defuzzification Methods using Application Specific Properties, IEEE Transactions on Fuzzy Systems, vol. 5, no. 1, pp. 72-79, 1997.

T. A. Runkler, M. Glesner, Defuzzification with Improved Static and Dynamic Behavior: Extended Center of Area, European Congress on Fuzzy and Intelligent Technologies, pp. 845-851, Aachen, Sept., 1993. 


\section{Appendix A: Connected Links}

\section{a) Current Links}

\begin{tabular}{|c|c|c|c|c|c|c|}
\hline Origin & Destination & Weight & Location 1 & Fuzzy 1 & Location 2 & Fuzzy 2 \\
\hline 1 & 3 & 39.2 & {$\left[\begin{array}{ll}10 & 9\end{array}\right]$} & 8.91 & {$\left[\begin{array}{ll}8 & 17\end{array}\right]$} & 7.98 \\
\hline 3 & 1 & 6.8 & {$\left[\begin{array}{ll}5 & 5\end{array}\right]$} & 1.6 & {$\left[\begin{array}{ll}0 & 10\end{array}\right]$} & 1.66 \\
\hline 2 & 3 & 30.4 & {$\left[\begin{array}{ll}10 & 8\end{array}\right]$} & 8 & {$\left[\begin{array}{ll}7 & 10\end{array}\right]$} & 7.22 \\
\hline 3 & 2 & 12.4 & {$\left[\begin{array}{ll}5 & 8\end{array}\right]$} & 4.65 & {$\left[\begin{array}{ll}5 & 7\end{array}\right]$} & 4.07 \\
\hline 3 & 4 & 36.8 & {$\left[\begin{array}{ll}10 & 6\end{array}\right]$} & 6.3 & {$\left[\begin{array}{ll}8 & 8\end{array}\right]$} & 7.46 \\
\hline 4 & 3 & 14.4 & {$\left[\begin{array}{ll}5 & 9\end{array}\right]$} & 5.01 & {$\left[\begin{array}{ll}5 & 8\end{array}\right]$} & 4.65 \\
\hline 4 & 5 & 15 & {$\left[\begin{array}{ll}5 & 10\end{array}\right]$} & 5.14 & [5 9] & 5.01 \\
\hline 5 & 4 & 10.4 & {$\left[\begin{array}{ll}5 & 6\end{array}\right]$} & 3.15 & {$\left[\begin{array}{ll}5 & 6\end{array}\right]$} & 3.15 \\
\hline 5 & 0 & 58.8 & {$\left[\begin{array}{ll}15 & 8\end{array}\right]$} & 12.9 & {$\left[\begin{array}{ll}12 & 10\end{array}\right]$} & 12.2 \\
\hline 0 & 5 & 46.3 & {$\left[\begin{array}{ll}10 & 16\end{array}\right]$} & 10.1 & {$\left[\begin{array}{ll}11 & 1 \\
0\end{array}\right]$} & 11.3 \\
\hline 0 & 6 & 93.2 & {$\left[\begin{array}{ll}20 & 20\end{array}\right]$} & 18.4 & {$\left[\begin{array}{ll}20 & 20\end{array}\right]$} & 18.4 \\
\hline 6 & 0 & 39.5 & {$\left[\begin{array}{ll}10 & 9\end{array}\right]$} & 8.91 & {$\left[\begin{array}{ll}8 & 17\end{array}\right]$} & 7.98 \\
\hline 6 & 7 & 26.3 & {$\left[\begin{array}{ll}6 & 14\end{array}\right]$} & 6.29 & {$\left[\begin{array}{ll}6 & 18\end{array}\right]$} & 6.29 \\
\hline 7 & 6 & 21.5 & {$\left[\begin{array}{ll}6 & 8\end{array}\right]$} & 5.92 & {$\left[\begin{array}{ll}6 & 8\end{array}\right]$} & 5.92 \\
\hline 7 & 8 & 14.8 & {$\left[\begin{array}{ll}5 & 10\end{array}\right]$} & 5.14 & {$\left[\begin{array}{ll}5 & 9\end{array}\right]$} & 5.01 \\
\hline 8 & 7 & 40 & {$\left[\begin{array}{ll}10 & 10\end{array}\right]$} & 10.1 & {$\left[\begin{array}{lll}8 & 18\end{array}\right]$} & 7.98 \\
\hline 0 & 9 & 20.8 & {$\left[\begin{array}{ll}6 & 7\end{array}\right]$} & 5.57 & {$\left[\begin{array}{ll}6 & 7\end{array}\right]$} & 5.57 \\
\hline 9 & 0 & 41 & {$\left[\begin{array}{ll}10 & 12\end{array}\right]$} & 10.1 & {$\left[\begin{array}{ll}8 & 19\end{array}\right]$} & 7.98 \\
\hline 9 & 23 & 46.7 & {$\left[\begin{array}{ll}10 & 16\end{array}\right]$} & 10.1 & {$\left[\begin{array}{ll}11 & 10\end{array}\right]$} & 11.3 \\
\hline 23 & 9 & 45 & {$\left[\begin{array}{ll}10 & 15\end{array}\right]$} & 10.1 & [11 9] & 10.1 \\
\hline 23 & 24 & 11.6 & {$\left[\begin{array}{ll}5 & 6\end{array}\right]$} & 3.15 & {$\left[\begin{array}{ll}5 & 7\end{array}\right]$} & 4.07 \\
\hline 24 & 23 & 28.2 & {$\left[\begin{array}{ll}6 & 16\end{array}\right]$} & 6.29 & {$\left[\begin{array}{ll}6 & 20\end{array}\right]$} & 6.29 \\
\hline 24 & 25 & 15.1 & {$\left[\begin{array}{ll}5 & 10\end{array}\right]$} & 5.14 & {$\left[\begin{array}{ll}5 & 9\end{array}\right]$} & 5.01 \\
\hline 25 & 24 & 40.1 & {$\left[\begin{array}{ll}10 & 10\end{array}\right]$} & 10.1 & {$\left[\begin{array}{ll}8 & 18\end{array}\right]$} & 7.98 \\
\hline 25 & 10 & 15 & {$\left[\begin{array}{ll}5 & 10\end{array}\right]$} & 5.14 & {$\left[\begin{array}{ll}5 & 9\end{array}\right]$} & 5.01 \\
\hline 10 & 25 & 40.1 & {$\left[\begin{array}{ll}10 & 10\end{array}\right]$} & 10.1 & {$\left[\begin{array}{lll}8 & 18\end{array}\right]$} & 7.98 \\
\hline 10 & 11 & 20.4 & [ 6 6 7] & 5.57 & {$\left[\begin{array}{ll}6 & 7\end{array}\right]$} & 5.57 \\
\hline 11 & 10 & 49.8 & {$\left[\begin{array}{ll}11 & 15\end{array}\right]$} & 11.3 & {$\left[\begin{array}{ll}11 & 13\end{array}\right]$} & 11.4 \\
\hline 0 & 12 & 67.5 & {$\left[\begin{array}{ll}15 & 17\end{array}\right]$} & 15 & {$\left[\begin{array}{ll}15 & 8\end{array}\right]$} & 12.9 \\
\hline 12 & 0 & 56.9 & {$\left[\begin{array}{lll}15 & 7\end{array}\right]$} & 12.1 & [12 9] & 10.9 \\
\hline 12 & 13 & 26.2 & {$\left[\begin{array}{ll}6 & 14\end{array}\right]$} & 6.29 & {$\left[\begin{array}{ll}6 & 18\end{array}\right]$} & 6.29 \\
\hline 13 & 12 & 26.2 & {$\left[\begin{array}{ll}6 & 14\end{array}\right]$} & 6.29 & {$\left[\begin{array}{ll}6 & 18\end{array}\right]$} & 6.29 \\
\hline 13 & 14 & 27.6 & {$\left[\begin{array}{ll}6 & 15\end{array}\right]$} & 6.29 & {$\left[\begin{array}{ll}6 & 19\end{array}\right]$} & 6.29 \\
\hline 14 & 13 & 32.8 & {$\left[\begin{array}{ll}10 & 6\end{array}\right]$} & 6.3 & {$\left[\begin{array}{ll}7 & 10\end{array}\right]$} & 7.22 \\
\hline 14 & 15 & 48 & {$\left[\begin{array}{ll}11 & 15\end{array}\right]$} & 11.3 & {$\left[\begin{array}{ll}11 & 13\end{array}\right]$} & 11.4 \\
\hline 15 & 14 & 67.5 & {$\left[\begin{array}{ll}15 & 17\end{array}\right]$} & 15 & {$\left[\begin{array}{ll}15 & 8\end{array}\right]$} & 12.9 \\
\hline 15 & 16 & 33.5 & {$\left[\begin{array}{ll}10 & 6\end{array}\right]$} & 6.3 & {$\left[\begin{array}{ll}7 & 10\end{array}\right]$} & 7.22 \\
\hline
\end{tabular}




\begin{tabular}{|r|r|r|r|r|r|r|}
16 & 15 & 69.8 & {$[1519]$} & 15 & {$[1510]$} & 15 \\
\hline 16 & 17 & 40.8 & {$[1011]$} & 10.1 & {$[819]$} & 7.98 \\
\hline 17 & 16 & 13.8 & {$[59]$} & 5.01 & {$[58]$} & 4.65 \\
\hline
\end{tabular}

\section{Appendix A: Connected Links (continued)}

\section{b) Alternative Links}

\begin{tabular}{|c|c|r|c|r|r|r|}
\hline Origin & Destination & Weight & Location 1 & Fuzzy 1 & Location 2 & Fuzzy 2 \\
\hline 1 & 18 & 11 & {$[56]$} & 3.15 & {$[57]$} & 4.07 \\
\hline 18 & 1 & 5 & {$[54]$} & 1.6 & {$[05]$} & 1.6 \\
\hline 2 & 18 & 10 & {$[56]$} & 3.15 & {$[56]$} & 3.15 \\
\hline 18 & 2 & 14 & {$[59]$} & 5.01 & {$[58]$} & 4.65 \\
\hline 3 & 15 & 2 & {$[02]$} & 1.6 & {$[00]$} & 1.6 \\
\hline 15 & 3 & 5 & {$[54]$} & 1.6 & {$[05]$} & 1.6 \\
\hline 4 & 12 & 9 & {$[56]$} & 3.15 & {$[55]$} & 1.6 \\
\hline 12 & 4 & 31 & {$[106]$} & 6.3 & {$[710]$} & 7.22 \\
\hline 5 & 12 & 27 & {$[615]$} & 6.29 & {$[619]$} & 10.9 \\
\hline 12 & 5 & 18 & {$[67]$} & 5.57 & {$[66]$} & 5.08 \\
\hline 4 & 19 & 12 & {$[59]$} & 5.01 & {$[57]$} & 4.07 \\
\hline 0 & 20 & 31 & {$[106]$} & 6.3 & {$[710]$} & 7.22 \\
\hline 20 & 6 & 8 & {$[56]$} & 3.15 & {$[55]$} & 1.6 \\
\hline 19 & 20 & 5 & {$[54]$} & 1.6 & {$[05]$} & 1.6 \\
\hline 20 & 19 & 0 & {$[00]$} & 1.6 & {$[00]$} & 1.6 \\
\hline 19 & 21 & 6 & {$[54]$} & 1.6 & {$[05]$} & 1.6 \\
\hline 6 & 21 & 5 & {$[54]$} & 1.6 & {$[05]$} & 1.6 \\
\hline 21 & 6 & 3 & {$[03]$} & 1.6 & {$[00]$} & 1.6 \\
\hline 21 & 7 & 11 & {$[56]$} & 3.15 & {$[57]$} & 4.07 \\
\hline 7 & 22 & 13 & {$[510]$} & 5.14 & {$[58]$} & 4.65 \\
\hline 6 & 9 & 41 & {$[1012]$} & 10.1 & {$[819]$} & 7.98 \\
\hline 22 & 23 & 18 & {$[67]$} & 5.57 & {$[66]$} & 5.08 \\
\hline 22 & 25 & 21 & {$[68]$} & 5.92 & {$[67]$} & 5.92 \\
\hline 9 & 13 & 17.2 & {$[67]$} & 5.57 & {$[66]$} & 5.08 \\
\hline 23 & 14 & 33 & {$[106]$} & 6.3 & {$[710]$} & 7.22 \\
\hline 24 & 15 & 21 & {$[68]$} & 5.92 & {$[67]$} & 5.92 \\
\hline 15 & 24 & 34 & {$[106]$} & 6.3 & {$[710]$} & 7.22 \\
\hline 25 & 27 & 25 & {$[614]$} & 6.29 & {$[615]$} & 6.29 \\
\hline 27 & 25 & 27 & {$[615]$} & 6.29 & {$[619]$} & 10.9 \\
\hline 26 & 27 & 5 & {$[54]$} & 1.6 & {$[05]$} & 1.6 \\
\hline 27 & 26 & 8 & {$[56]$} & 3.15 & {$[55]$} & 1.6 \\
\hline 15 & 26 & 10 & {$[56]$} & 3.15 & {$[56]$} & 3.15 \\
\hline & & & & & & \\
\hline
\end{tabular}




\begin{tabular}{|l|r|r|r|r|r|r|}
26 & 15 & 7 & {$[55]$} & 1.6 & {$[05]$} & 1.6 \\
\hline 26 & 16 & 14.4 & {$[59]$} & 5.01 & {$[58]$} & 4.65 \\
\hline 16 & 26 & 7.36 & {$[55]$} & 1.6 & {$[55]$} & 1.6 \\
\hline
\end{tabular}

\section{Appendix B: Results of Experiment using Two Parameters (Demand and Distance)}

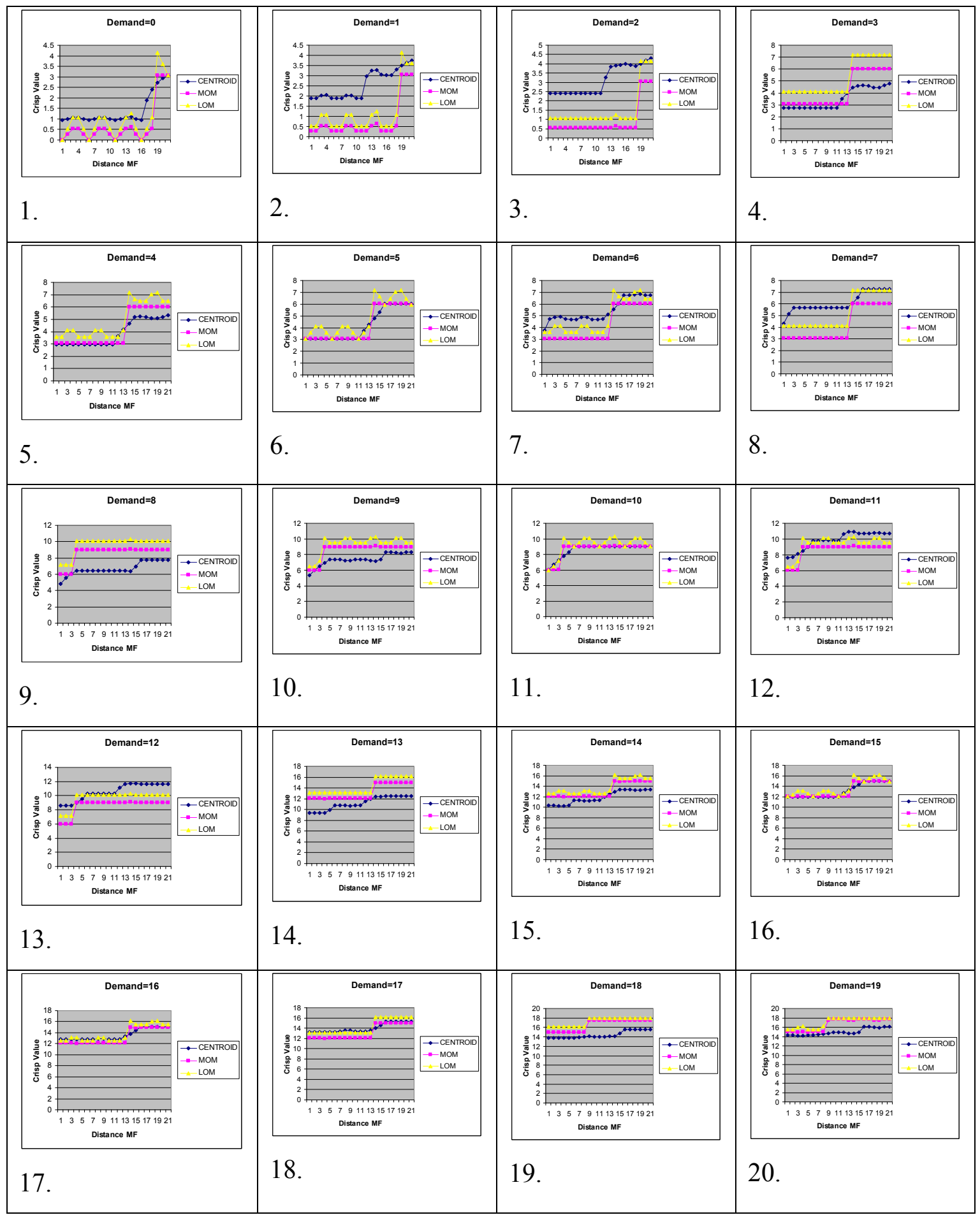




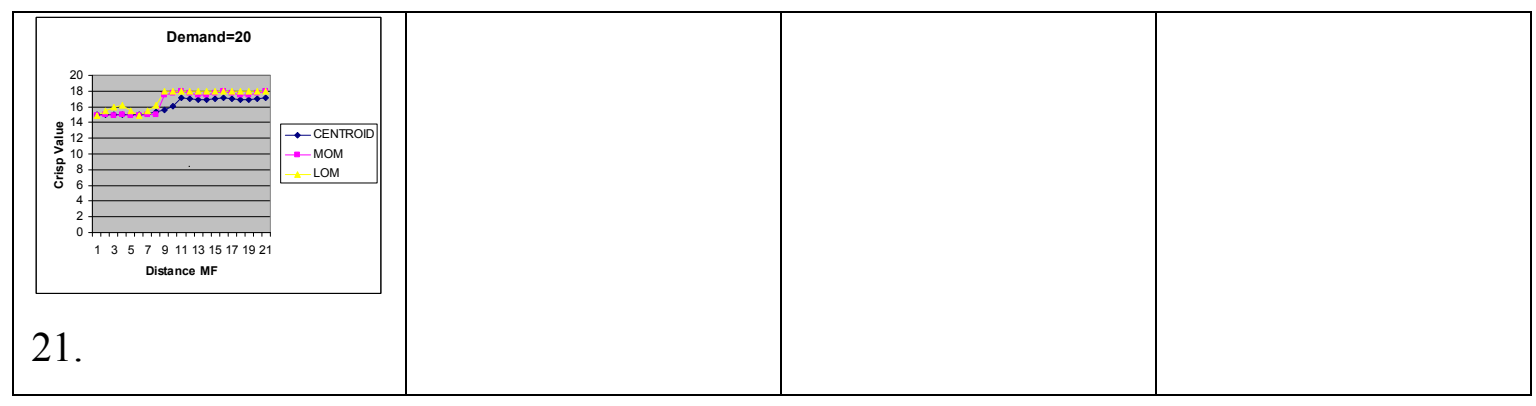

\section{Appendix C: Results of Experiment using Three Parameters (Demand, Distance, and Type of Road)}

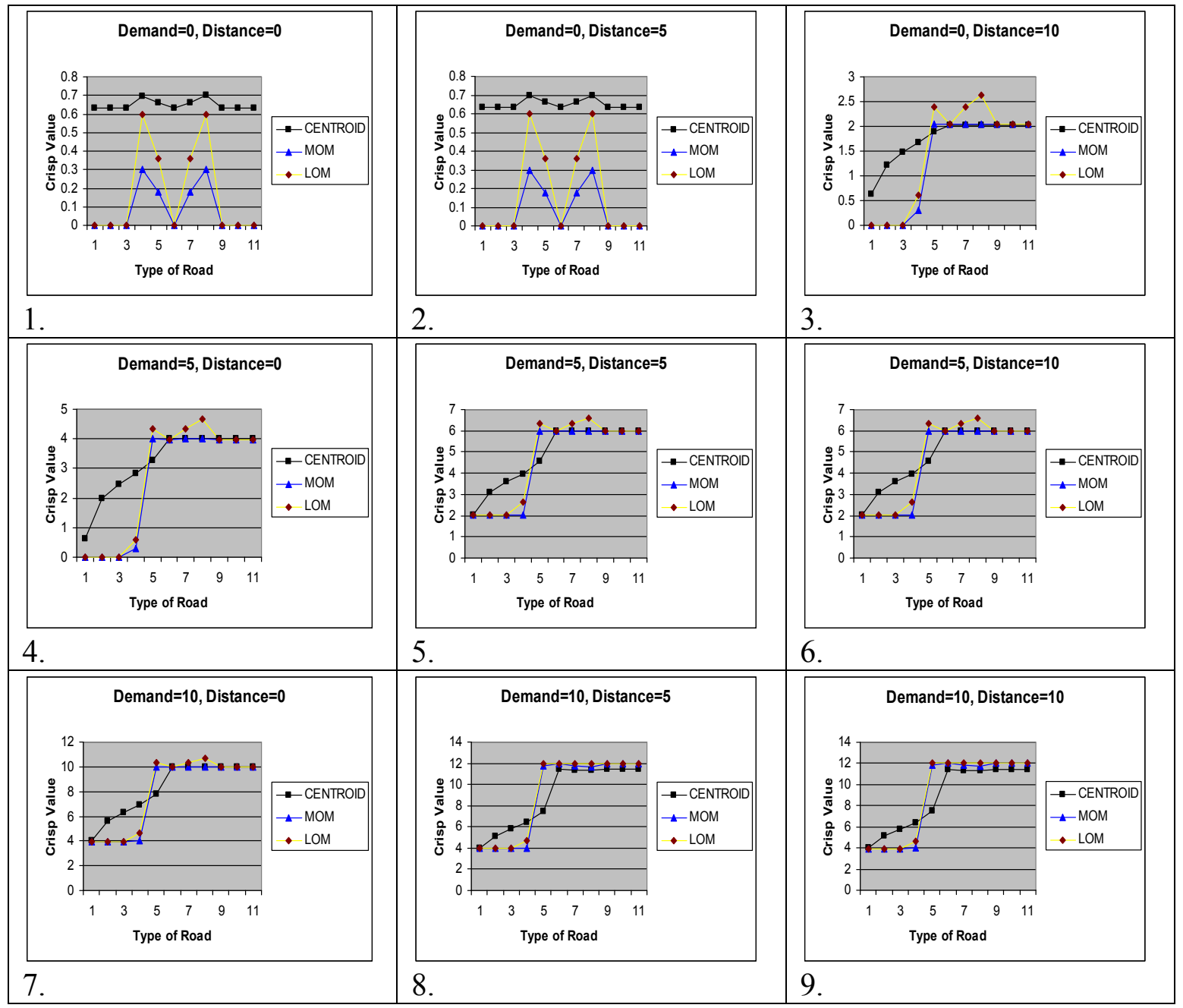

